\title{
A SUBQUADRATIC ALGORITHM FOR COMPUTING THE $n$-TH BERNOULLI NUMBER
}

\author{
DAVID HARVEY
}

ABstract. We describe a new algorithm that computes the $n$th Bernoulli number in $n^{4 / 3+o(1)}$ bit operations. This improves on previous algorithms that had complexity $n^{2+o(1)}$.

\section{INTRODUCTION}

The Bernoulli numbers $B_{0}, B_{1}, B_{2}, \ldots$ are rational numbers defined by

$$
\frac{t}{e^{t}-1}=\sum_{k=0}^{\infty} \frac{B_{k}}{k !} t^{k} \text {. }
$$

Every odd-index Bernoulli number is zero except for $B_{1}=-1 / 2$. The von StaudtClausen theorem states that the denominator of $B_{2 n}$ is precisely the product of the primes $p$ such that $p-1 \mid 2 n$, and Euler's formula

$$
B_{2 n}=(-1)^{n+1} \frac{2(2 n) !}{(2 \pi)^{2 n}} \zeta(2 n),
$$

where $\zeta(s)=\sum_{k=1}^{\infty} k^{-s}$ is the Riemann zeta function, implies that the number of bits in the numerator of $B_{2 n}$ is $\Theta(n \log n)$.

It is known that the first $n$ Bernoulli numbers may be computed simultaneously in $n^{2} \log ^{2+o(1)} n$ bit operations [BH11. This bound is optimal up to logarithmic factors, as the total number of bits being computed is $\Theta\left(n^{2} \log n\right)$. (In this paper, "bit operations" always means number of operations in the multitape Turing model, as in Pap94.)

The situation concerning computation of a single $B_{n}$ is less satisfactory. As $B_{n}$ has $n^{1+o(1)}$ bits, it is conceivable that it can be computed in only $n^{1+o(1)}$ bit operations. However, the best published complexity bounds have the shape $n^{2+o(1)}$, which is essentially no better than computing all of $B_{0}, \ldots, B_{n}$. This is achieved by two quite different algorithms: the "zeta function algorithm", which approximates $\zeta(2 n)$ in the right-hand side of (1.1) via the Euler product (this has been rediscovered numerous times — see the discussion in [Har10]), and the multimodular algorithm introduced by the author in Har10.

In this paper we close two thirds of this gap. Our main result is:

Theorem 1.1. Let $\frac{1}{3} \leq \alpha \leq \frac{1}{2}$. The Bernoulli number $B_{n}$ may be computed in

$$
n^{1+\alpha} \log ^{4-\alpha+o(1)} n
$$

Received by the editor October 14, 2012 and, in revised form, February 7, 2013.

2010 Mathematics Subject Classification. Primary 11B68, 11Y55.

The author was supported by the Australian Research Council, DECRA Grant DE120101293. 
bit operations, using

$$
O\left(n^{2-2 \alpha} \log ^{1+2 \alpha} n\right)
$$

bits of space.

The bounds are uniform in $\alpha$, i.e., the implied $O(\cdot)$ constant does not depend on $\alpha$, and the $o(1)$ term approaches zero as $n \rightarrow \infty$, independently of $\alpha$.

In particular, taking $\alpha=1 / 3$, we obtain the time bound

$$
n^{4 / 3} \log ^{11 / 3+o(1)} n
$$

and space bound $O\left(n^{4 / 3} \log ^{5 / 3} n\right)$. The parameter $\alpha$ permits a time-space tradeoff. Taking $\alpha=1 / 2$ increases the time to $n^{3 / 2} \log ^{7 / 2+o(1)}$, but reduces the space usage to $O\left(n \log ^{2} n\right)$. In this latter case, further logarithmic savings in time and space may be achieved; see Remark 3.7

Our strategy may be summarised as follows. It is technically convenient to work with the Genocchi numbers [Com74, p. 49] given by

$$
G_{n}=2\left(1-2^{n}\right) B_{n} .
$$

It follows immediately from the von Staudt-Clausen theorem and Fermat's little theorem that $G_{n} \in \mathbf{Z}$. Moreover, $\log G_{n}=O(n \log n)$, so asymptotically the number of bits we must determine is the same as for $B_{n}$.

In [Har10], we gave a formula, sometimes known as a Voronoi congruence, that expresses $B_{n}(\bmod p)$ as a sum of $O(p)$ terms, for a prime $p$. Evaluating this formula for sufficiently many $p$, and combining the results using the Chinese remainder theorem, led to the overall complexity bound $n^{2+o(1)}$ for computing $B_{n}$.

Proposition 2.1 below may be interpreted as a generalisation of this formula from a congruence modulo $p$ to a congruence modulo $p^{s}$, expressing $G_{n}\left(\bmod p^{s}\right)$ as a sum of $O(p s)$ terms. Evaluating this formula in the straightforward way has complexity $O\left(p s^{2}\right)$ (ignoring logarithmic factors), because we are doing arithmetic in $\mathbf{Z} / p^{s} \mathbf{Z}$, whose elements have $O(s \log p)$ bits. This approach has more flexibility than that of Har10, as we may choose $s$ as a function of $p$ to optimise the total cost. Unfortunately, it turns out that this still leads to a quasi-quadratic complexity bound for computing $B_{n}$.

However, we make two key observations. First, provided $p$ is not too small compared to $s$, we can use techniques of fast polynomial arithmetic to save a factor of roughly $s$ in the evaluation of the formula of Proposition 2.1. This trick is already enough to lower the overall cost to $n^{3 / 2+o(1)}$ (see Remark 3.7). Second, by some algebraic rearrangement and careful choice of coefficient rings, we may evaluate the formula of Proposition 2.1 for many primes simultaneously. This reduces the complexity further to $n^{4 / 3+o(1)}$.

We do not know for what $n$ an efficient implementation of these new algorithms would be faster than existing implementations of the quasi-quadratic algorithms. This is an interesting question for further study.

\section{Congruences}

Proposition 2.1. Let $n \geq s \geq 1$ and let $p$ be an odd prime. Let

$$
F_{p}(x)=\sum_{k=0}^{s-1}\left(\begin{array}{c}
n \\
k+1
\end{array}\right) G_{k+1} p^{k} x^{s-1-k} \in \mathbf{Z}[x] .
$$


Then

$$
G_{n}=\sum_{j=0}^{p-1}(-1)^{j} j^{n-s} F_{p}(j) \quad\left(\bmod p^{s}\right) .
$$

Proof. Our proof is modelled on [Coh07, Prop. 9.1.3].

The exponential generating function for the $G_{n}$ is

$$
\sum_{n \geq 0} \frac{G_{n}}{n !} t^{n}=\sum_{n \geq 0} \frac{2 B_{n}}{n !} t^{n}-\sum_{n \geq 0} \frac{2 B_{n}}{n !}(2 t)^{n}=\frac{2 t}{e^{t}-1}-\frac{4 t}{e^{2 t}-1}=\frac{2 t}{e^{t}+1} .
$$

The Genocchi polynomials

$$
G_{n}(x)=\sum_{k=0}^{n}\left(\begin{array}{l}
n \\
k
\end{array}\right) G_{k} x^{n-k} \in \mathbf{Z}[x]
$$

have exponential generating function given by

$$
E(t, x)=\sum_{n \geq 0} \frac{G_{n}(x)}{n !} t^{n}=\left(\sum_{k \geq 0} \frac{G_{k}}{k !} t^{k}\right)\left(\sum_{m \geq 0} \frac{x^{m}}{m !} t^{m}\right)=\frac{2 t e^{t x}}{e^{t}+1} .
$$

Now on one hand we have

$$
\sum_{j=0}^{p-1}(-1)^{j} E(p t, j / p)=\sum_{n \geq 0} \frac{p^{n}}{n !} \sum_{j=0}^{p-1}(-1)^{j} G_{n}(j / p) t^{n},
$$

while on the other hand this sum is also equal to

$$
\sum_{j=0}^{p-1}(-1)^{j} \frac{2 p t e^{t j}}{e^{p t}+1}=\frac{2 p t}{e^{p t}+1} \sum_{j=0}^{p-1}\left(-e^{t}\right)^{j}=\frac{2 p t}{e^{t}+1}=p \sum_{n \geq 0} \frac{G_{n}}{n !} t^{n} .
$$

Equating coefficients of $t^{n}$ and using $G_{0}=0$ we obtain

$$
G_{n}=p^{n-1} \sum_{j=0}^{p-1}(-1)^{j} G_{n}(j / p)=\sum_{j=0}^{p-1}(-1)^{j} \sum_{k=0}^{n-1}\left(\begin{array}{c}
n \\
k+1
\end{array}\right) G_{k+1} j^{n-k-1} p^{k} .
$$

Truncating this sum modulo $p^{s}$ yields the desired congruence.

\section{Algorithms}

Let $n \geq s \geq 1$, and define

$$
F(x)=\sum_{k=0}^{s-1}\left(\begin{array}{c}
n \\
k+1
\end{array}\right) G_{k+1} x^{s-1-k} \in \mathbf{Z}[x] .
$$

Note that $F(x)$ depends on $n$ and $s$, but (crucially) not on $p$. For any prime $p \geq 3$ and any $0 \leq j<p$ we have $F_{p}(j)=p^{s-1} F(j / p)$. We obtain the following bounds for the coefficients of $F(x)$ and for $F_{p}(j)$.

Lemma 3.1. Let $n \geq 1$ and $0 \leq k<n$. Then

$$
\left|\left(\begin{array}{c}
n \\
k+1
\end{array}\right) G_{k+1}\right| \leq 7(n / \pi)^{k+1} \text {. }
$$


Proof. For $k=0$ the assertion is that $n \leq 7(n / \pi)$. For even $k \geq 2$, we have $G_{k+1}=0$. For odd $1 \leq k<n$, by (1.1) we have

$$
\begin{aligned}
\left|\left(\begin{array}{c}
n \\
k+1
\end{array}\right) G_{k+1}\right| & =\frac{n !}{(k+1) !(n-k-1) !} 2\left(2^{k+1}-1\right) \frac{2(k+1) !}{(2 \pi)^{k+1}} \zeta(k+1) \\
& \leq 4 \zeta(2) \frac{n !}{(n-k-1) !} \frac{1}{\pi^{k+1}} \leq 6.579 \ldots(n / \pi)^{k+1} .
\end{aligned}
$$

Lemma 3.2. Let $n \geq s \geq 4$. Let $p$ be an odd prime and let $0 \leq j<p$. Then

$$
\left|F_{p}(j)\right| \leq 3(n p / \pi)^{s+1} \text {. }
$$

Proof. By the previous lemma we have

$$
\begin{aligned}
\left|F_{p}(j)\right| & \leq \sum_{k=0}^{s-1} 7(n / \pi)^{k+1} p^{k} j^{s-1-k} \leq 7 p^{s-1} \sum_{k=0}^{s-1}(n / \pi)^{k+1}=7 p^{s-1}(n / \pi) \frac{(n / \pi)^{s}-1}{n / \pi-1} \\
& \leq \frac{7}{3^{2}(4 / \pi-1)}(n p / \pi)^{s+1}=2.846 \ldots(n p / \pi)^{s+1} .
\end{aligned}
$$

We recall some standard results concerning the complexity of integer and polynomial arithmetic; all of this may be found in vzGG03.

Let $R=\mathbf{Z} / 2^{M} \mathbf{Z}$ where $M \geq 1$. Addition and subtraction in $R$ require $O(M)$ bit operations. Multiplication in $R$ costs $M \log ^{1+o(1)} M$ bit operations, using $O(M)$ bits of space, via FFT methods. Division in $R$ (where possible) has the same asymptotic time and space complexity as multiplication, using Newton's method. If $G \in R[x]$ is a polynomial of degree $s$, and $x_{1}, \ldots, x_{t} \in R$, with $t \leq s$, then we may simultaneously evaluate $G\left(x_{1}\right), \ldots, G\left(x_{t}\right) \in R$ using a fast multipoint evaluation algorithm in $s M \log ^{1+o(1)}(s M) \log s$ bit operations. The simplest such algorithms have space complexity $O(s M \log s)$, but this can be reduced to $O(s M)$ by the method of [vzGS92, Lemma 2.1].

Now let $p$ be an odd prime, $s \geq 1$, and $R=\mathbf{Z} / p^{s} \mathbf{Z}$. We assume here that $p \leq n$ and $s \leq n$. The results are similar: addition and subtraction in $R$ require $O(s \log p)=O(s \log n)$ bit operations, and multiplication in $R \operatorname{costs} s \log ^{2+o(1)} n$ bit operations.

Finally, we mention that the primes $p \leq N$ may be enumerated by a straightforward sieve method in $N^{1+o(1)}$ bit operations.

Proposition 3.3. Let $n \geq s \geq 4$ and let $N \leq n$. Let $P$ be a set of primes with $3 \leq p<N$ for all $p \in P$. Assume that $\sum_{p \in P} p \leq s$. Then the residues $G_{n}$ $\left(\bmod p^{s}\right)$ may be computed for all $p \in P$ simultaneously in

$$
s^{2} \log ^{3+o(1)} n
$$

bit operations, using

$$
O\left(s^{2} \log n\right)
$$

bits of space.

Proof. Let $M=\left\lceil\log _{2}\left(3(n N / \pi)^{s+1}\right)\right\rceil+1$. For this choice of $M$, by Lemma 3.2 we have $\left|F_{p}(j)\right|<2^{M} / 2$ for all $p \in P, 0 \leq j<p$, so to compute $F_{p}(j)$ it suffices to determine it modulo $2^{M}$. Note that $M=O(s \log n)$.

We perform the following steps, each of which uses $O\left(s^{2} \log n\right)$ space. 
Step 1. Compute $G_{k}$ for $1 \leq k \leq s$ using (for example) the algorithm of [BH11]. This costs $s^{2} \log ^{2+o(1)} s=s^{2} \log ^{2+o(1)} n$ bit operations.

Step 2. Compute $\left(\begin{array}{l}n \\ k\end{array}\right)$ for $1 \leq k \leq s$. Using a straightforward algorithm this can be done in $O\left(s^{2} \log ^{2} n\right)$ bit operations.

Step 3. Compute the coefficients of $F(x)$, by computing the products $\left(\begin{array}{l}n \\ k\end{array}\right) G_{k}$ for $0 \leq k \leq s$. Each product needs $s \log ^{2+o(1)} n$ bit operations. The total cost is $s^{2} \log ^{2+\bar{o}(1)} n$ bit operations.

Step 4. Compute $j / p\left(\bmod 2^{M}\right)$ for each $p \in P, 0 \leq j<p$. Each division costs $M \log ^{1+o(1)} M=s \log ^{2+o(1)} n$ bit operations. Since we have assumed that $\sum_{p \in P} p \leq s$, the total cost is $s^{2} \log ^{2+o(1)} n$ bit operations.

Step 5. Regarding $F(x)$ as a polynomial in $\left(\mathbf{Z} / 2^{M} \mathbf{Z}\right)[x]$, evaluate simultaneously $F(j / p)\left(\bmod 2^{M}\right)$ for all $p \in P, 0 \leq j<p$. This costs $s^{2} \log ^{3+o(1)} n$ bit operations.

Step 6. For each $p \in P, 0 \leq j<p$, recover $F_{p}(j)=p^{s-1} F(j / p)\left(\bmod 2^{M}\right)$, and hence the exact integer $F_{p}(j)$. Since $p^{s-1} \leq 2^{M}$, we may compute $p^{s-1}$, and then $F_{p}(j)$, in $M \log ^{1+o(1)} M=s \log ^{2+o(1)} n$ bit operations, and thus the total cost is $s^{2} \log ^{2+o(1)} n$ bit operations.

Step 7. For each $p \in P, 0 \leq j<p$, compute $j^{n-s}\left(\bmod p^{s}\right)$. Each power costs $(\log n)\left(s \log ^{2+o(1)} n\right)=s \log ^{3+o(1)} n$ bit operations, so the total cost is $s^{2} \log ^{3+o(1)} n$ bit operations.

Step 8. Use Proposition 2.1 to recover $G_{n}\left(\bmod p^{s}\right)$ for each $p \in P$. The cost is $s^{2} \log ^{2+o(1)} n$ bit operations.

Remark 3.4. The complexity of Step 7 can be improved, by computing first $q^{n-s}$ $\left(\bmod p^{s}\right)$ for primes $q<p$, and then using $\left(j_{1} j_{2}\right)^{n-s}=j_{1}^{n-s} j_{2}^{n-s}$ for composite $j=j_{1} j_{2}$. This saves a factor of $\log n$ in this step, provided that $p$ is not too small, say $p>n^{c}$ for any fixed $c>0$. This will be the case for almost all primes $p$ used in the proof of Theorem 1.1 .

Remark 3.5. In a practical setting, one may wish to replace the $\operatorname{ring} \mathbf{Z} / 2^{M} \mathbf{Z}$ by $\mathbf{Z} / T \mathbf{Z}$ where $T$ is a suitably large integer not divisible by any $p \in P$. For example, one could take $T$ to be a product of many word-sized primes $q$ for which there exist efficient number-theoretic transforms modulo $q$. Under this scheme, the expensive evaluation in Step 5 could be performed for each $q$ separately, and then the $F_{p}(j)$ could be reconstructed in Step 6 using the Chinese remainder theorem. This approach does not change the asymptotic complexity, but potentially yields a drastic improvement in memory locality.

Remark 3.6. Further practical savings may be realised by using the easily-proved fact that $G_{n}(1-x)=-G_{n}(x)$ for even $n$, so that

$$
G_{n}=2 \sum_{j=1}^{(p-1) / 2}(-1)^{j} j^{n-s} F_{p}(j) \quad\left(\bmod p^{s}\right) .
$$

Coupled with the observation that essentially half of the coefficients of $F(x)$ are zero, this leads to a savings of a factor of two in the main evaluation step.

Now we may prove the main result. 
Proof of Theorem 1.1. Recall that $1 / 3 \leq \alpha \leq 1 / 2$. We will take

$$
N=\left\lfloor n^{\alpha} \log ^{1-\alpha} n\right\rfloor, \quad s=\left\lfloor 2 n^{1-\alpha} \log ^{\alpha} n\right\rfloor .
$$

We may assume that $n$ is large enough so that $n \geq s \geq N \geq 4$. In particular, we may assume that the hypotheses of Proposition 3.3 are satisfied.

Let $P$ be the set of odd primes $p<N$, so that $|P|=O(N / \log N)=O\left(n^{\alpha} \log ^{-\alpha} n\right)$. Let $r=\left\lfloor 2 n^{1-2 \alpha} \log ^{2 \alpha-1} n\right\rfloor$. Note that $r \geq 1$ for sufficiently large $n$. Partition $P$ into $d$ sets $P_{1}, \ldots, P_{d}$ of cardinality at most $r$, where $d=O(|P| / r)=$ $O\left(n^{3 \alpha-1} \log ^{1-3 \alpha} n\right)$. For each $i$ we have $\sum_{p \in P_{i}} p \leq\left|P_{i}\right| N \leq r N \leq s$.

Apply Proposition 3.3 to each set $P_{i}$ separately. The space usage for each invocation is $O\left(s^{2} \log n\right)=O\left(n^{2-2 \alpha} \log ^{2 \alpha+1} n\right)$. This space may be reused for each $P_{i}$. The total time cost is $d s^{2} \log ^{3+o(1)} n=n^{1+\alpha} \log ^{4-\alpha+o(1)} n$.

At this stage we have computed $G_{n}\left(\bmod p^{s}\right)$ for all $p \in P$. This is enough to determine $G_{n}$ (for sufficiently large $n$ ), because

$$
\log \prod_{p \in P} p^{s}=s \sum_{3 \leq p<N} \log p \sim s N=2 n \log n+O(n),
$$

whereas $\log G_{n}=n \log n+O(n)$. Using fast Chinese remaindering we may then recover $G_{n}$, and hence $B_{n}$, in $n^{1+o(1)}$ bit operations.

Remark 3.7. We sketch an algorithm that improves the time and space complexities, respectively, to $n^{3 / 2} \log ^{3+o(1)} n$ and $O(n \log n)$ in the case $\alpha=1 / 2$. Consider the algorithm of Proposition 3.3 applied to a set $P=\{p\}$ consisting of a single prime. The evaluation points $j / p$, for $0 \leq j<p$, now form an arithmetic progression. We relax the condition $p \leq s$, instead allowing $p$ as large as $s \log s$. Instead of evaluating at all $p$ points simultaneously, we first evaluate at only $s$ points, and then use the value-shifting algorithm of [Sho91, Theorem 3.1] (alternatively the algorithm of [BGS07, Theorem 5]) to evaluate at the remaining $p-s$ points, in blocks of $s$ points at a time. Then in the proof of Theorem 1.1 we take $s=\left\lfloor 2 n^{1 / 2}\right\rfloor$ and $N=\left\lfloor n^{1 / 2} \log n\right\rfloor$, and only use Proposition 3.3 for one prime at a time. This leads to the complexity bounds stated above; we omit the proof, which is similar to that of Theorem 1.1

\section{ACKNOWLEDGMENTS}

Many thanks to Joe Buhler, Bernd Kellner and an anonymous referee for their comments on a draft of this paper.

\section{REFERENCES}

[BGS07] Alin Bostan, Pierrick Gaudry, and Éric Schost, Linear recurrences with polynomial coefficients and application to integer factorization and Cartier-Manin operator, SIAM J. Comput. 36 (2007), no. 6, 1777-1806, DOI 10.1137/S0097539704443793. MR2299425 (2008a:11156)

[BH11] Richard P. Brent and David Harvey, Fast computation of Bernoulli, Tangent and Secant numbers, in Proceedings of a Workshop on Computational and Analytical Mathematics in honour of Jonathan Borwein's 60th birthday, Springer Proceedings in Mathematics, Vol. 50, 2013, 127-142, http://dx.doi.org/10.1007/978-1-4614-7621-4_8.

[Coh07] Henri Cohen, Number theory. Vol. II. Analytic and modern tools, Graduate Texts in Mathematics, vol. 240, Springer, New York, 2007. MR.2312338 (2008e:11002)

[Com74] Louis Comtet, Advanced combinatorics, Revised and enlarged edition, D. Reidel Publishing Co., Dordrecht, 1974. The art of finite and infinite expansions. MR.0460128 (57 \#124) 
[Har10] David Harvey, A multimodular algorithm for computing Bernoulli numbers, Math. Comp. 79 (2010), no. 272, 2361-2370, DOI 10.1090/S0025-5718-2010-02367-1. MR:2684369 (2011h:11019)

[Pap94] Christos H. Papadimitriou, Computational complexity, Addison-Wesley Publishing Company, Reading, MA, 1994. MR1251285 (95f:68082)

[Sho91] Victor Shoup, A fast deterministic algorithm for factoring polynomials over finite fields of small characteristic, Proceedings of the 1991 International Symposium on Symbolic and Algebraic Computation (New York, NY, USA), ISSAC '91, ACM, 1991, pp. 14-21.

[vzGG03] Joachim von zur Gathen and Jürgen Gerhard, Modern computer algebra, 2nd ed., Cambridge University Press, Cambridge, 2003. MR2001757 (2004g:68202)

[vzGS92] Joachim von zur Gathen and Victor Shoup, Computing Frobenius maps and factoring polynomials, Comput. Complexity 2 (1992), no. 3, 187-224, DOI 10.1007/BF01272074. MR:1220071 (94d:12011)

School of Mathematics and Statistics, University of New South Wales, Sydney NSW 2052, Australia

E-mail address: d.harvey@unsw.edu.au 